\title{
Journalof the optical Society

\section{Visual and physiological optics: introduction to the Journal of the Optical Society of America A feature issue}

\author{
Harilaos Ginis, ${ }^{1, *}$ Vasyl Molebny, ${ }^{2}$ Jos J. Rozema, ${ }^{3}$ Christina Schwarz, ${ }^{4}$ and Juan Tabernero ${ }^{5}$ \\ ${ }^{1}$ Athens Eye Hospital, Greece \\ ${ }^{2}$ Institute of Biomedical Engineering, Ukraine \\ ${ }^{3}$ University of Antwerp, Belgium \\ ${ }^{4}$ University of Tübingen, Germany \\ ${ }^{5}$ Anglia Ruskin University, UK \\ *Corresponding author: h.ginis@athenseyehospital.gr
}

Received 28 March 2019; posted 28 March 2019 (Doc. ID 363684); published 1 April 2019

\begin{abstract}
The 9th European Meeting on Visual Physiological Optics (VPO2018) was held August 29-31, 2018, in Athens, Greece. This issue of the Journal of the Optical Society of America A (JOSA A) is a dedicated feature, including numerous articles that span a large range of visual optics related topics, ranging from geometrical optics to visual psychophysics and from optical metrology to ophthalmic diagnostic technologies. The next VPO meeting will be held August 2020 in Cambridge, UK. @ 2019 Optical Society of America
\end{abstract}

https://doi.org/10.1364/JOSAA.36.00VPO1

Visual and Physiological Optics is at present one of the fields that are defined by the applications rather than the applicable methods or the associated technology. About 150 years ago a great period of fast discovery started, during which scientists documented in detail the eye's optical system. In parallel, optical instruments to study the eye were developed. Understanding the eye and developing the technologies for studying or treating the eye have always progressed in parallel. This course of parallel development continues today as new features in the eye are revealed by two-photon and Optical Coherence Tomography imaging techniques and new treatments are delivered by femtosecond lasers. The early devices were developed mainly by ophthalmologists seeking practical solutions to their everyday clinical needs for diagnosis and treatment. These were the likes of von Helmholtz, Donders, and Gullstrand, who were highly trained in physics and mathematics, and literally wrote the book on visual optics. Today, in many aspects we are in a situation where the technology has surpassed clinical application. Various new diagnostic devices provide data with, at this moment, unclear value for clinical decision making, and various treatment modalities represent solutions beyond our solid understanding of the patient's needs. As an example, we have hundreds of different intraocular lens designs for cataract surgery, as well as precise knowledge of the retinal image associated with each design, but we have no solid methodology to decide which lens is best suited for each individual patient. The next step in this succession of parallel development seems to be into improving (again) our understanding of the eye and human vision: Understanding peripheral vision and its role in quality of life, linking objective optical characteristics and functional vision, raising the bar for the definition of successful treatments, and specifying real-world applications for the exciting technologies that are still in the research laboratories.

The VPO meetings have always served the link between basic science and the clinical world. The upcoming VPO 2020 in Cambridge, UK may reflect the next step in Visual and Physiological Optics: improving vision. 